\title{
CONSEJO COMUNAL DE INFANCIA DE ANTOFAGASTA: HISTORIA Y TESTIMONIOS DE UNA EXPERIENCIA PIONERA DE REPRESENTACIÓN Y PARTICIPACIÓN INFANTIL
}

Communal Council of Infancy of Antofagasta: history and testimonies of a pioneer experience of representation and child participation

Conselho Comunitário de Infantia de Antofagasta: história e testemunhos de uma experiência pioneira de representação e participação de crianças

\author{
Marco Alvarez Parra \\ Universidad de Valparaíso, Chile \\ marcoalvarezparra@gmail.com \\ Katherin Castillo Morales \\ Universidad Católica del Norte, Chile \\ kscastillom@gmail.com
}

\begin{abstract}
Resumen
El Consejo Comunal de Infancia de la comuna de Antofagasta en sus 8 años de existencia ha formado y servido de plataforma de participación infantil para niños, niñas y adolescentes de la comuna de Antofagasta. El presente artículo busca sistematizar esta experiencia en el relato de una historia y sus testimonios, conocer el funcionamiento de esta instancia, los logros que ha obtenido y por sobretodo la experiencia que ha significado este órgano de representación y praxis infantil para los niños y niñas consejeros de infancia de la comuna de Antofagasta. Para esto se trabajó una metodología cualitativa en base a la técnica de entrevista semiestructurada, el análisis de documentos institucionales y del registro de las actividades más importantes realizadas en el transcurso de la historia del consejo.

Palabras Clave: participación infantil; ciudadanía; Consejo Comunal de Infancia Antofagasta
\end{abstract}




\begin{abstract}
The Communal Council of Infancy of the commune of Antofagasta in its 8 years of existence has formed and served as a platform of child participation for children and adolescents of the commune of Antofagasta. The present article seeks to systematize this experience in the story of a story and its testimonies, to know the functioning of this instance, the achievements it has obtained and above all the experience that this body of representation and child praxis has meant for the boys and girls counselors of childhood of the commune of Antofagasta. For this, a qualitative methodology was used based on the semi-structured interview technique, the analysis of institutional documents and the registry of the most important activities carried out in the course of the council's history.
\end{abstract}

Key words: children's participation; citizenship; Communal Childhood Council Antofagasta

\title{
Resumo
}

O Conselho Comunal da Infância da comuna de Antofagasta, em seus 8 anos de existência, formou-se e serviu de plataforma de participação infantil para crianças e adolescentes da comuna de Antofagasta. O presente artigo busca sistematizar essa experiência na história de uma história e seus depoimentos, conhecer o funcionamento desta instância, as conquistas obtidas e, sobretudo, a experiência que esse corpo de representação e práxis infantil significou para os conselheiros de meninos e meninas de infância da comuna de Antofagasta. Para isso, utilizou-se uma metodologia qualitativa baseada na técnica de entrevista semiestruturada, na análise de documentos institucionais e no registro das atividades mais importantes realizadas no decorrer da história do conselho. Palavras-chave: participação infantil; cidadania; Conselho Comunal de Infância Antofagasta

\section{Introducción y presentación de la experiencia}

Si bien la Convención de los Derechos del Niño posiciona a la Participación Infantil como un derecho reflejado en la libertad de opinión, de expresión, de pensamiento y de asociación, se le debe considerar también como una condición indispensable para el mejoramiento real de la situación de vida de niños, niñas y adolescentes ya que la participación al ocurrir 
fomenta el despliegue de las capacidades de la niñez en los aspectos más variados de su vida (Marquez; 2009).

En este contexto el presente artículo busca presentar la experiencia del Consejo Comunal de Infancia de Antofagasta, instancia que a sus 8 años de existencia, ha visto pasar a más de 150 niños, niñas y adolescentes de la comuna quienes en su desempeño de Consejeros de Infancia han trabajado con sus pares y el mundo adulto logrando impactar e incidir de distintas formas en la construcción de una comuna más amigable con la infancia, y donde ellos mismos se posicionan como actores y agentes relevantes en la construcción de la ciudad. Se quiso mostrar esta experiencia desde la voz de los propios actores, por lo que en términos metodológicos, y desde una perspectiva cualitativa, se profundizó en el discurso y los significados de los entrevistados/as. En correspondencia con lo anterior se utilizó la técnica de entrevista semiestructurada y el análisis de documentos institucionales y del registro de las actividades más importantes realizadas en el transcurso de la historia del consejo.

Con todo, creemos importante visibilizar este sistemático trabajo que se ha realizado por los niños y niñas consejeros/as de infancia, que han formado parte de este órgano de representación infantil, y a la vez, que estos sean de ejemplo para las próximas generaciones que luchen por el derecho a una ciudad donde los niños, niñas y adolescentes sean actores considerados, tomados en cuenta y acogidos.

\subsection{Consejo Comunal de Infancia de Antofagasta}

Fundado durante el año 2010, el Consejo Comunal de Infancia (CCI) de Antofagasta, se ha transformado en un espacio vital para la participación infantil y adolescente en la comuna de Antofagasta. Siendo impulsado por la Ilustre Municipalidad de Antofagasta, el CCI busca ser una instancia de reunión, discusión, praxis y colaboración entre los niños, niñas y adolescentes (NNA), con el objetivo de impulsar la participación del mundo infantoadolescente en la comuna con las distintas autoridades comunales, poder incidir en las decisiones que se tomen sobre ellos y ellas y de promover la generación de una Política Comunal de Infancia que reconozca a todo NNA como sujeto de derecho. 
Esta iniciativa busca cumplir con los estándares del programa "Ciudades Amigas para la Infancia" de Unicef, promoviendo la democratización de las decisiones que se toman en la ciudad en temas relativos a la infancia, o en propuestas que surgen de los niños, niñas y jóvenes para mejorar, a través de canales municipales, la calidad de vida de la población infanto-juvenil.

Es importante señalar que el consejo lo componen NNA de los distintos establecimientos educacionales de la comuna, como también actores con necesidades educativas especiales y/o no insertos en el sistema escolar. De esta manera ellos trabajan de forma colaborativa con autoridades y actores relevantes de la ciudad, en la elaboración y desarrollo de soluciones en torno a las problemáticas que les afecten, tales como han sido el alcoholismo, la drogadicción, el caso callejero, el embarazo adolescente, el bullyng, la contaminación, por nombrar algunas temáticas, las cuales son elegidas de forma democrática por los mismos consejeros en el Encuentro de Participación Infantil que la Casa Comunal de Infancia convoca año a año.

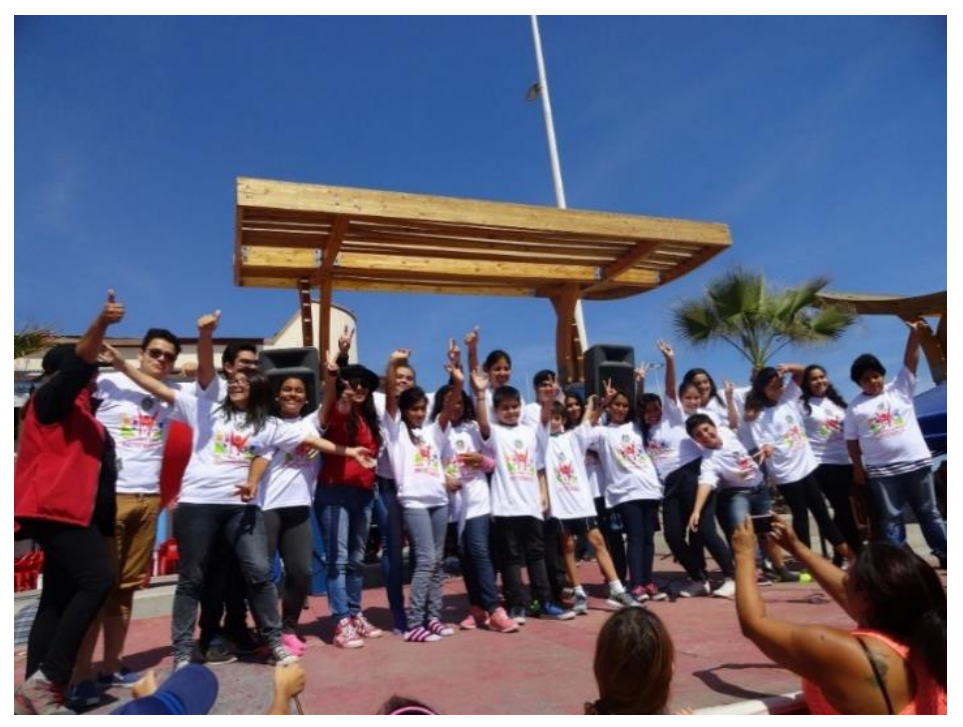

Imagen 1. Fuente: elaboración propia

\subsection{El Consejo Comunal de Infancia de Antofagasta: áreas de acción y temáticas} abordadas

Desde los primeros años de existencia los Consejeros de Infancia han seleccionado y trabajado diversas temáticas a través de grupos de trabajo y comisiones conformadas de 
común acuerdo entre ellos mismos. En este sentido Diego Cayupi, ex consejero del Consejo Comunal de Infancia (CCI) en 2009, señala que en un principio se abordaron temáticas tales como la alimentación, la relación con la JUNAEB, el bullyng y los espacios públicos para el esparcimiento, la recreación y el deporte en relación a la infancia de la comuna. Dichas problemáticas fueron en un principio tratadas en las distintas comisiones y reuniones de coordinación del CCI, para ser luego elevadas a una discusión con los miembros del Concejo Municipal y de esta manera poder llevar ante las autoridades locales las inquietudes y demandas de los NNA de la comuna.

Por su parte, Francisco Pastene, señala que la metodología de las reuniones fundacionales tanto como las sostenidas a lo largo del año no distaban mucho de las actuales, las cuales son de índole participativas, convocando de forma abierta a todos los niños y niñas de la comuna, donde entre 2011 y 2012 se tematizaron las cuestiones relativas al Espacio Público (medio ambiente, la recreación y la integración).

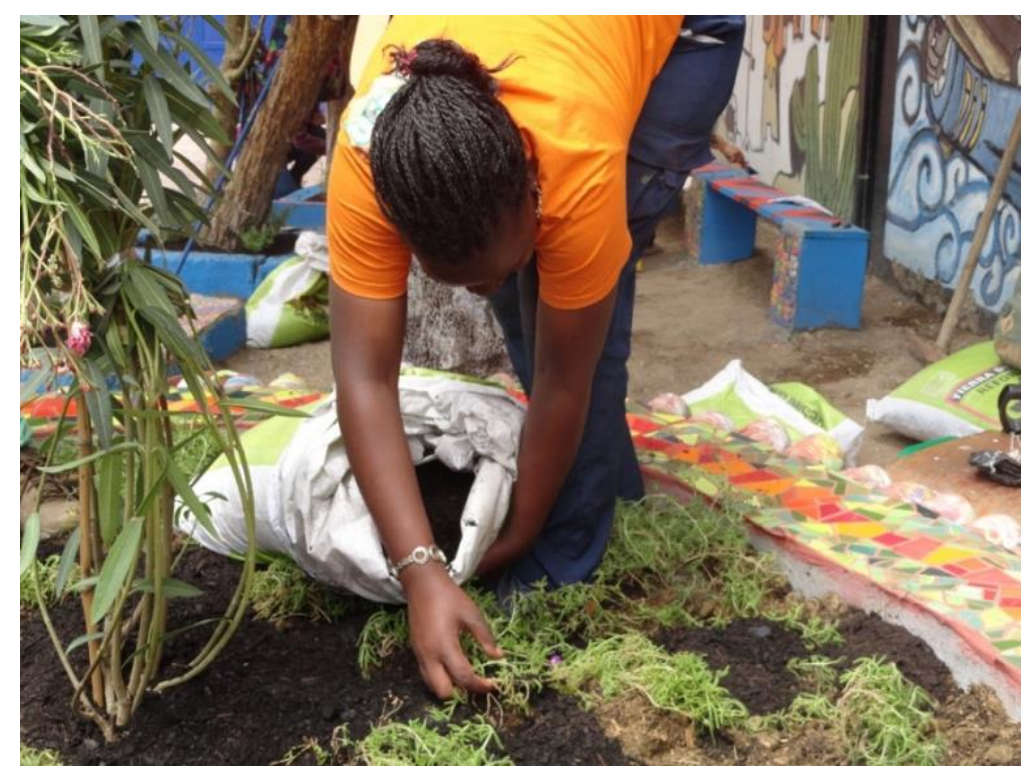

Imagen 2. Fuente: elaboración propia

En la etapa de desarrollo del CCI entre 2013 y 2014, se constata el trabajo de las temáticas de áreas verdes, tránsito, e inclusión y convivencia escolar, representadas por las comisiones integradas por los consejeros. En ese contexto, el tema de la decisión sobre el espacio público ocupa un lugar privilegiado a la hora de hablar de los problemas y cómo éstos fueron resueltos 
por el actuar del CCI. Constatar aquel hecho sirve para dar cuenta de cómo los problemas del espacio público tienen un papel protagónico en la discusión de los intereses que tiene la infancia y juventud en la ciudad, al igual que los problemas relativos al reconocimiento de las diferentes identidades (como es el caso de jóvenes con discapacidades psico-motoras, personas con orientaciones sexuales LGTB o comunidad migrante) en el seno de las comunidades educativas.

El espacio de asamblea del CCI sirve como el contexto idóneo para que los niños, niñas y jóvenes puedan discutir y socializar sus intereses en comunidad para finalmente elegir qué temáticas abordar. En vista de que el fomento a la participación entre semejantes permite la interacción de las distintas experiencias que tienen en su espacio educativo y comunidad local, atendiendo a discutir lo que quieren para el espacio vinculante que es en último término la comuna, los niños, niñas y jóvenes han podido desarrollar competencias que formalmente no ocupan un lugar en el currículum educativo, así como tienen la oportunidad de conocer experiencias de vida que no forman parte de su cotidianeidad, como puede ocurrir con la presencia de estudiantes de distintos niveles socioeconómicos o lugares de la ciudad.

Por otro lado se valora el funcionamiento del consejo y el cómo se implementan sus decisiones, su periodicidad y su carácter orgánico-formal apareciendo este último elemento como el de mayor importancia siempre considerando el contexto emergente de crecientes transformaciones vividas por la ciudad de Antofagasta.Las áreas trabajadas por el consejo tendrían también una estricta relación con la

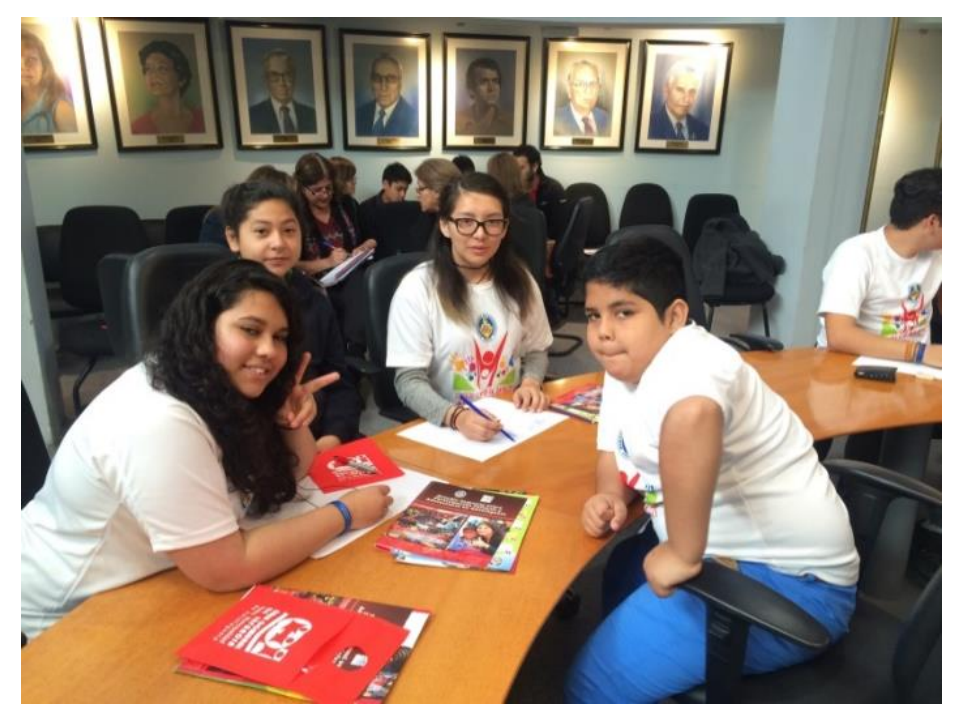
forma en la que los miembros del consejo van incorporando sus visiones de mundo y también la problematización de su realidad que realizan tocados por sus proyectos educativos. La confluencia de los miembros en el consejo no versa exclusivamente sobre sus experiencias 
personales a nivel familiar, sino está también empapada de los proyectos educativos como de los distintos énfasis marcados por las comunidades educativas.

En lo que respecta al proceso de consolidación del CCI, se destaca la existencia de 3 comisiones desde 2016 hasta abril de 2017: Acoso Callejero, Mal Uso de Redes Sociales y abuso de alcohol y drogas, seleccionadas por los Consejeros de Infancia a partir de las temáticas problematizadas en los Encuentros de Participación Infantil donde se convoca a las niñas y niños de la comuna de manera abierta. Dicha instancia es particularmente importante en la medida que sirve para la definición e identificación colectiva de los intereses de los asistentes, quienes provienen de los distintos establecimientos educacionales de la comuna.

Quizás un rasgo distintivo de dicho proceso de consolidación 2016-2017 puede tener que ver con el hecho de que las temáticas problematizadas por los miembros dicen mucha mayor relación con lo que es la contingencia del país, y no tanto con las problemáticas específicas que podían apreciar en concreto en su propio entorno. Dicha cuestión, volcó el trabajo del CCI hacia un trabajo de despliegue territorial en los establecimientos educacionales de la comuna enfocando el esfuerzo de las comisiones en la concientización, educación y debate en torno a temáticas que son de carácter nacional y no sólo local, al contrario de años anteriores donde las

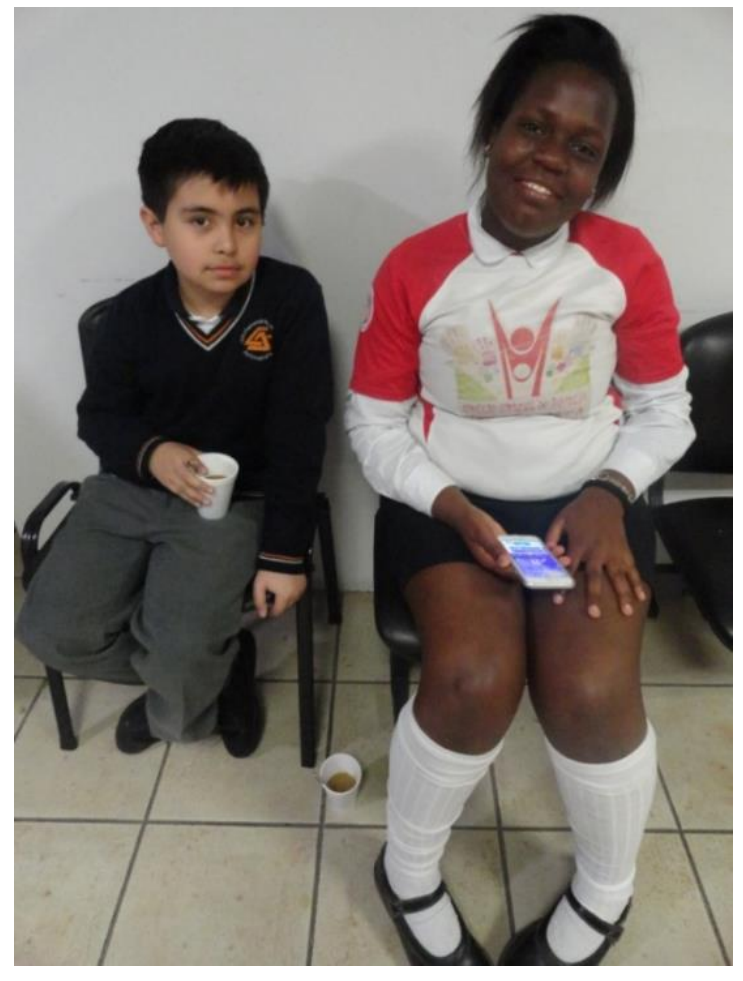
comisiones levantadas por el Consejo priorizaron problemáticas de la comuna que llevaron al CCI incluso a tener presencia en los Plenos Municipales, es decir, a trabajar directamente con las autoridades y no directamente con los niños y niñas y adolescentes de la comuna. 
Con todo podemos señalar que ambos niveles de trabajo, tanto territorial-educacional como a nivel de debate político, son relevantes en la consecución de logros significativos para el CCI, y por ende, para la población infanto-adolescente de la comuna, que por un lado necesita educarse, sensibilizarce y capacitarse respecto a ciertas temáticas que les afectan pero que por otro lado requiere ser escuchada y considerada en las decisiones que toman, respecto a diversas temáticas, las autoridades comunales.

Sin duda los resultados que dichas acciones puedan tener a futuro merecen la expectación no sólo por parte de las autoridades y comunidades locales, y especialmente de quienes se vean directamente afectados en éstas por los temas en cuestión, sino de toda la región y el país, en busca de precedentes significativos para emular el trabajo en las distintas comunidades territoriales y para el potencial diseño de políticas públicas que permitan el tratamiento democrático de dichas cuestiones.

\section{Principales logros obtenidos}

Los principales logros obtenidos para los consejeros no son identificados como realizaciones abstractas, sino con el logro de metas concretas, como por ejemplo, la construcción del parque Gran Avenida como un nuevo pulmón verde el cual está ubicado en el sector norte de Antofagasta, el proyecto de restauración del Parque Brasil, las Becas Municipales de excelencia académica o el Skatepark Pablo Neruda. Un lugar importante en el discurso de los consejeros es el ocupado por las actividades de sensibilización a la comunidad infantoadolescente, destacando entre ellas la Expo Interactiva de Infancia y la participación del CCI en Recreos Interactivos donde, de acuerdo a la temática trabajada, se sensibilizaba y educaba a sus pares de forma lúdica y llamativa a través de los distintos establecimientos de la comuna. También destacan dentro de estas actividades el seminario "Abriendo espacios a las Infancias y Juventudes de Antofagasta” el cual se realizó el presente año siendo organizado por los mismos Consejeros de Infancia quienes luego de un importante trabajo de capacitación con distintas entidades a lo largo del año, concientizaron y educaron a niños, niñas y adolescentes antofagastinos sobre las temáticas trabajadas en una jornada donde las comisiones expusieron de manera interactiva las diversas temáticas escogidas. 
En términos de Política Local, a partir de la experiencia de los niños y niñas consejeros de infancia en torno a la violencia de género y los problemas de convivencia intercultural en los estudiantes de la comuna, se impulsaron dos convenios: Convenio contra la Violencia en el Pololeo y Convenio por la Convivencia Intercultural, donde se realizó un trabajo intersectorial entre el Consejo de Infancia, la Municipalidad de Antofagasta y la Corporación Municipal de Desarrollo Social (CMDS Educación), e instituciones tales como SERNAMEG, Servicio Jesuita a Migrantes, entre otras, con la finalidad de comprometer un trabajo sistemático de educación y sensibilización en los establecimiento educacionales de la comuna, teniendo como resultado la realización de más de 1500 estudiantes sensibilizados y 200 profesionales psicosociales capacitados en la temática Violencia en el Pololeo. Por su parte desde el convenio de convivencia intercultural se realizó un importante trabajo de sensibilización en 4 establecimientos educacionales de la comuna donde de forma sistemática se impulsó el programa Escuelas Interculturales en Antofagasta, desde el cual se trabajó con estudiantes, profesores, apoderados y directivos de los distintos establecimientos a lo largo de todo el año 2017. Estos dos, importantes logros obtenidos desde el CCI.

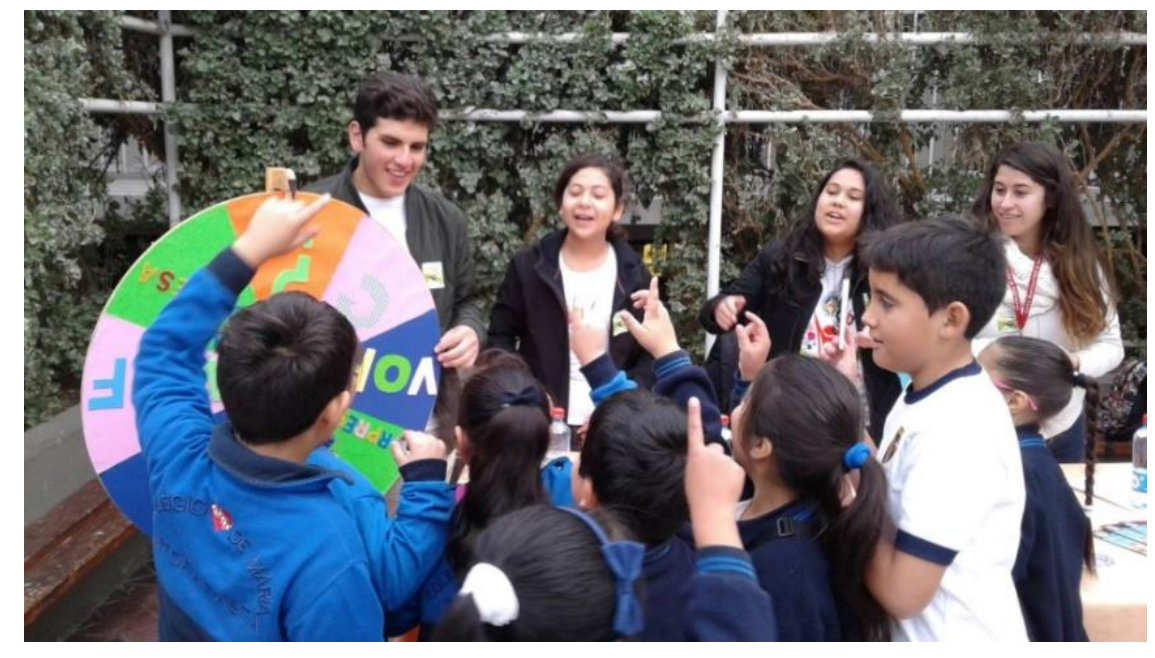

Sumado a lo anterior, los consejeros valoran de distinta manera los logros obtenidos, por ejemplo, para el consejero Marcial Valdivia, un importante logro fue que el CCI pudo ser "la voz de los sin voz", pudiendo ser escuchados por las autoridades o bien lo señalado por el consejero Manuel del Canto, quien destaca como fundamental la visibilización de los niños no sólo en la gestión local, sino que en todo el proceso de creación de la políticas públicas 
municipales. Por otra parte se identifica un sentimiento de satisfacción e identificación de los consejeros respecto a los logros en materia de infraestructura y espacios públicos, como por ejemplo el Parque Brasil:
"Me pueden decir "ya, el parque Brasil está bien". Pero el parque Brasil lo hicimos nosotros, el parque Brasil lo coordinamos nosotros." (Tobar, exconsejero)

Además de este logro, todos los demás exconsejeros coinciden en destacar el papel jugado por los miembros que integraron el CCI en la planificación y ejecución del proyecto para el Skatepark, no solo como consecución de un producto concreto del trabajo llevado a cabo por el CCI, sino también como una iniciativa que permitió la inclusión activa de los miembros más jóvenes que lo integraban, como señalan Pastene y Tobar, y con un apoyo concitado por los demás actores (como los profesionales de la Casa Comunal de Infancia).

A su vez, es un consenso general entre consejeros y exconsejeros considerar como un fruto el hecho de que el trabajo en conjunto haya permitido la obtención de los logros hacia la comunidad, con un especial énfasis dirigido a consejeros provenientes de instituciones noeducativas como es el caso de Teletón y el Hogar de Cristo. Dichos miembros han tenido la oportunidad de ser parte de una organización de participación infantil que de manera constante potencia sus capacidades y demanda de ellos diversas responsabilidades al asumir el rol de Consejero o Consejera de Infancia. Cabe mencionar que también se realizaron mejoras en equipamiento de los espacios públicos como es el caso de la instalación de juegos inclusivos, lo que da también cuenta del carácter integrador de dicho logro.

Otro de los logros del CCI identificados es la participación vinculada a la representación de la diversidad social que habita Antofagasta en los términos más formales posibles. El consejero Marcial Valdivia ilustra de manera bastante clara cómo se inicia regularmente el trabajo que sostienen los miembros del consejo de cara al año:

"En los Encuentros de participación se busca entonces recoger la mayor parte de las problemáticas que aquejan a las comunidades, en conjunto con sus representantes." (Marcial Valdivia, consejero vigente). 
Justamente, uno de los principales logros de dicha instancia es la generación de un espacio de convocatoria abierta a la infancia de la comuna de Antofagasta en el que se decidan participativamente los elementos que la comunidad infanto-adolescente abordará a lo largo del año, así como la posibilidad de que los representantes de la comunidad puedan otorgar a lo largo del año la retroalimentación de lo ocurrido en el CCI a éstas mismas.

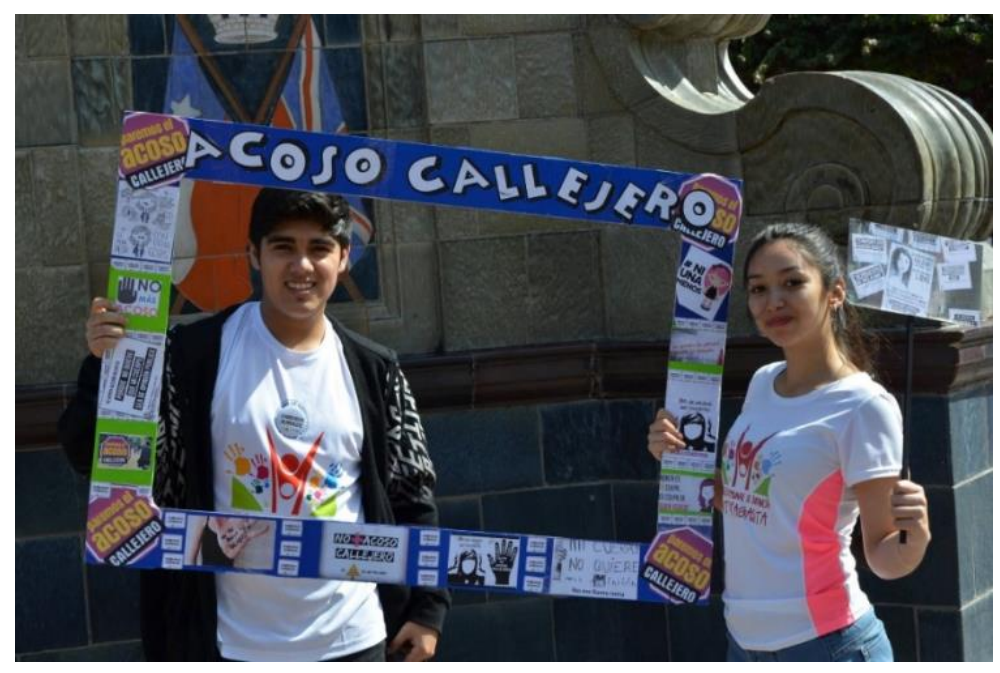

Asimismo, se destaca el problema que significa una potencial disonancia entre los intereses de los consejeros y los programas municipales, motivo que produjo inquietudes en los consejeros con respecto a los logros en su trabajo en el Consejo. Dicho esto, los profesionales se sienten satisfechos con el hecho de que los cambios en el trabajo desarrollado por los consejeros han sido sugeridos y valorado por los propios miembros de éste, quizás sin lograr realizar todas las iniciativas concretas que han esbozado.

Eso deja de lado el hecho de que se han tenido productos concretos como es el caso del nuevo Pulmón verde de Antofagasta o las ya nombradas ferias interactivas, sólo que el éxito de dichas cuestiones pareciera relativizar el papel del CCI como impulsor de iniciativas, frente a la voluntad política del Concejo Municipal. En ese sentido, se considera que el elemento que más facilitó el éxito fue la buena voluntad de la administración de abrir los espacios locales a la posibilidad de injerencia del Consejo, y la posibilidad de mantener dichas vinculaciones a lo largo del tiempo. 
En términos generales, es posible dar cuenta de que el principal logro del CCI es ofrecer una posibilidad concreta para que los jóvenes tengan un espacio permanente en que tomen una posición hacia su realidad y hacia sus comunidades sobre las problemáticas que les aquejan. Asimismo, es posible establecer que el aval de parte de las instituciones educativas, como el apoyo brindado por los profesionales de la Casa Comunal y la comunicación con la Municipalidad es indispensable en la consagración de condiciones apropiadas para la obtención de logros al corto y mediano plazo, que estimulen la proactividad y la problematización de los elementos que conforman el día a día de los consejeros y los espacios que representan, mayormente instituciones educativas, junto al Hogar de Cristo y Teletón.

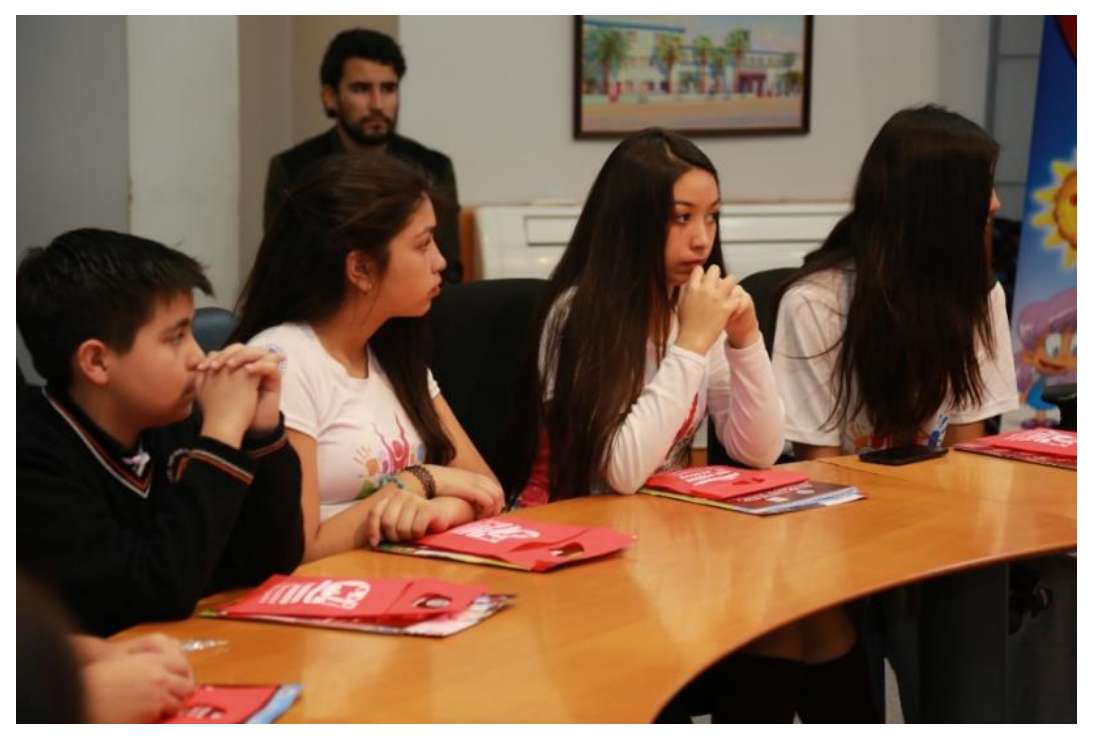

Lo anterior permite afirmar el hecho de que el trabajo llevado a cabo por el Consejo ha logrado conciliar el llevar a cabo una discusión y reflexión permanente sobre el cómo se viven los problemas de la contingencia nacional, así como también cómo se ve afectada la vida de las comunidades territoriales locales por sus problemas particulares y por el papel que juega Antofagasta en la región y el país.

Sin duda que los consejeros/as se agrupen, se organicen y desafíen sus propias competencias y sus capacidades para trabajar en grupo se traduce en un ejercicio colectivo permanente de reflexión sobre sí mismos, sus comunidades, su educación y la sociedad. 
En este punto, la formación de líderes se entiende como una tarea colectiva que involucra un aprendizaje permanente, que compromete activamente a los consejeros no sólo en la obtención de logros concretos o de realizaciones abstractas, sino en entramar una práctica cotidiana de liderazgo dirigida al desarrollo y entrada en diálogo de sus comunidades con otras a través del consejo, y de comprometer junto con ellos a compañeros representados

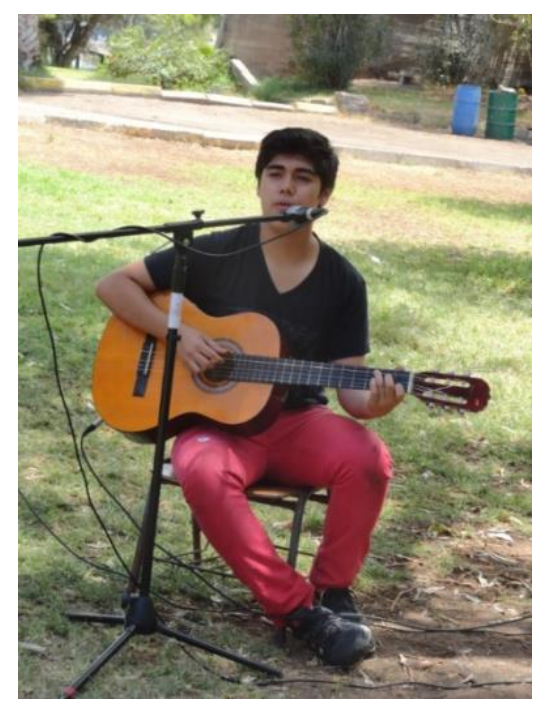
como comunidades que intervienen activamente en lo que ocurra en su comuna.

La tematización de las distintas aristas abordadas por el trabajo de las comisiones, y cómo los actores entrevistados han descrito que se ha realizado aquel trabajo, ha señalado con mayor énfasis el hecho de entender a los distintos actores, presentes de una u otra manera en el CCI, como parte de la misma labor de constitución del nosotros comunitario, y ha ofrecido la posibilidad de realización de derechos ciudadanos para todos quienes se ven beneficiados de una u otra manera por el Consejo.

\section{La experiencia del consejo: los cambios a través de la participación}

Para la consecución de los logros en cuestión, los consejeros y exconsejeros señalan que los mayores facilitadores de procesos son la buena comunicación y cercanía con las autoridades como el Concejo Municipal o la alcaldía y la legitimidad que poseen, siendo instancias que han ofrecido buena acogida a las iniciativas del CCI, y a que estas logren una buena recepción en las comunidades.

El consejero vigente Manuel del Canto valora especialmente el hecho de que la formación entregada por la participación permanente en el consejo no trata exclusivamente sobre los temas tratados en comisión, sino que en el desarrollo de habilidades para llegar a las personas y abordar problemas colectivamente.

En el caso de Marcial Valdivia, éste destaca su manejo de habilidades blandas como unas herramienta de posicionamiento como dirigente, ocupando roles de vocería y secretaría. Sin 
embargo, desestima el valor que tienen dichas posiciones por relevar el papel que debe jugar el consejo como instancia de representación y participación. Manuel del Canto, por su parte, asume desde su trayectoria y papeles jugados en el consejo una posición similar. Ambos coinciden en que el CCI les ha brindado un espacio para su desarrollo individual, así como para su crecimiento personal y la toma de una mayor conciencia de sus problemas como de los problemas que aquejan a sus comunidades.

Matías Tobar, por otro lado, señala que la posibilidad de ser escuchado por las autoridades es el cambio fundamental que ha generado la instancia conformada por el CCI y los canales propios del municipio. Personalmente, es también capaz de verse a sí mismo como un aporte significativo al CCI, y viendo a este último como un aporte significativo para su conocimiento y desplante en público, ofreciendo un testimonio bastante parecido al de los dos jóvenes antes citados.

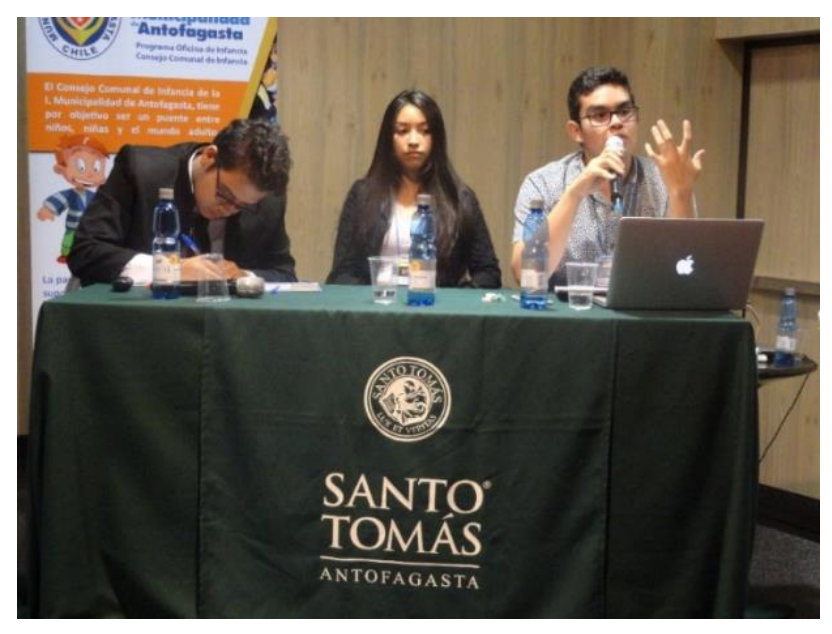

El ex consejero Pastene, por su parte, señala que su experiencia en los dos años en los que participó del consejo le permitió especialmente abrirse a la necesidad del trabajo en equipo y generar redes a futuro con la Municipalidad, resultando especialmente enriquecedora para su formación como estudiante secundario, así como útil estando ya en la universidad. En el Consejo supo conocer la preocupación por el que no resultaran las cosas, así como también vivir una experiencia de construcción hacia su comunidad marcada por el ambiente fraternal vivido con los distintos miembros del consejo. 
En el caso de la ex consejera Daniela Pumarino, cabe dar cuenta de dos hechos muy importantes que marcaron su experiencia, como es el hecho de enfrentarse a un espacio mayormente conformado por hombres jóvenes, y que a la vez, eran casi todos mayores que ella. A partir de su temprana inserción en el espacio, Daniela se ganó un lugar entre sus compañeros, a la vez que sirvió para generar un ambiente que motivara su desempeño en la agrupación. La ex consejera describe su participación como una cuestión muy positiva, pudiendo crecer personalmente y superar los problemas típicos de la juventud que la aquejaban en su entorno más cercano:

"Me sacaron del hoyito que yo estaba y me hicieron florecer en ese aspecto, en realidad era tímida y guagüita que era, salí una niña con personalidad, con entusiasmo, motivación (...) formé más mi carácter, formé mucho mi carácter, adquirí más personalidad, perdí mucha vergüenza, porque igual era como medio complicado que te pasaron el micrófono y hablarle a un auditorio lleno de niños, profesores, era distinto a disertar en la sala de clases me ayudó mucho en eso, en la personalidad, la vergüenza, el carácter, esos fueron como los atributos que más me dieron.” (Daniela Pumarino, ex consejera).

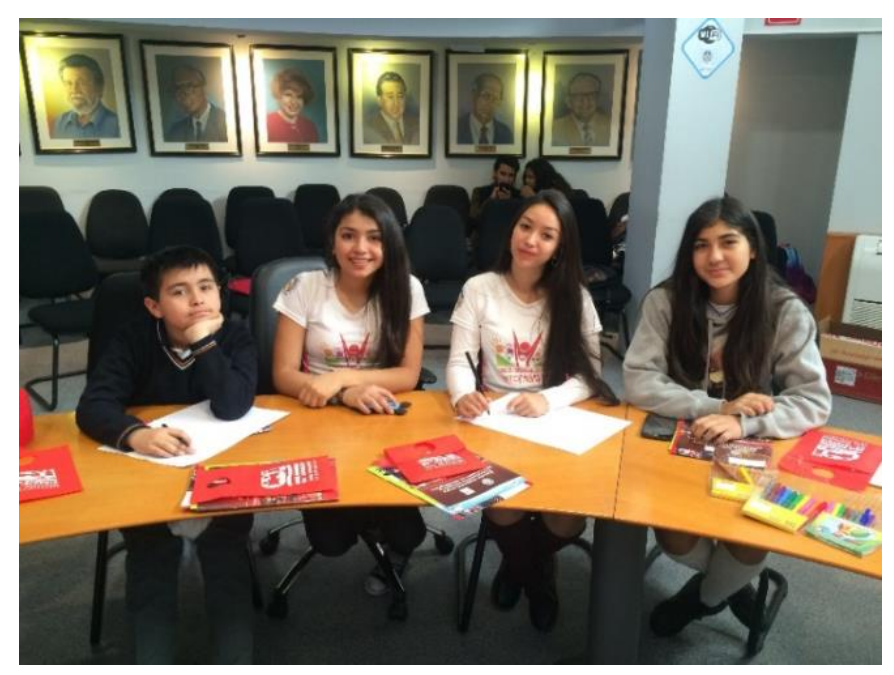

Diego Cayupi, primer vocero, y luego vicevocero del CCI, afirma que el Consejo le sirvió para poder dilucidar cuánto podía aportar a la sociedad. Para él fue una experiencia que califica como fantástica, dado que no sólo abrió sus ojos a la realidad que ocurría fuera de su entorno más cercano, sino que le entregó las herramientas para empoderarse en dicha realidad 
y participar activamente de ella. Esto, evidentemente, no queda exento de la generación de vínculos de amistad con los distintos miembros que conformaban el CCI en su época, con los que se impulsó aún más las iniciativas que vieron nacer al consejo.

Podemos afirmar, en lo que respecta a la experiencia de participación de los jóvenes en el CCI, que este es un espacio que entrega más preguntas que certezas, pero que ofrece la garantía de poder resolver dichas preguntas acorde a un buen trabajo en conjunto, y a la coordinación con las personas e instituciones capacitadas para llevar a cabo los distintos cambios que comprometen la participación en sociedad de los integrantes del Consejo. Asimismo, existe también un consenso generalizado en torno a la utilidad tanto práctica como valórica que significa el ejercicio de una democracia construida por y para ellos mismos como consejeros.

Cabe dar cuenta del hecho de que se entrevista a jóvenes que han ocupado distintas posiciones al interior del Consejo, pero que de todas formas han ocupado dicho espacio como una ventana hacia el mundo de la misma manera que como una ventana a sí mismos, pudiendo fortalecer su autoconciencia y entregando parte de su experiencia como aprendizaje para las próximas generaciones de líderes en formación.

\section{Percepciones: la valoración del Consejo}

Los consejeros identifican el CCI como una instancia para la formación de un liderazgo político orientado a la apertura de espacios de participación y comunicación con las comunidades y sus problemáticas. En general, se percibe el CCI como una organización activa y fructífera para el desempeño de quienes lo integran. Frente a lo anterior, el Consejero Marcial Valdivia señala:

"En Antofagasta y Chile actualmente no hay instancias legales de participación ciudadana infantil y juvenil, la única instancia de participación ciudadana en estos momentos son los Consejos Comunales de Infancia que están operando a lo largo de Chile". (Marcial Valdivia, consejero vigente) 
Dicha afirmación es respaldada por el Consejero Manuel del Canto a partir de la percepción que el Consejo tiene tanto de su participación como importante, así como del papel que el propio consejo tiene ante las autoridades.

Se asimila el aseguramiento de la mayor representatividad posible con la presencia de niños, niñas y jóvenes que no forman necesariamente parte de la escolaridad regular. Esto, dando cuenta de una percepción integral y solidaria de la niñez y la juventud por parte de los consejeros, que ven como pares no a todo aquel que cuente con la posibilidad formal de tener la misma educación que ellos reciben o podrían recibir, sino a quienes viven y deben enfrentarse a problemas similares propios de su edad, ocupan un papel similar en sus familias y en sus territorios, y tienen intereses confluentes. Uno de los mejores ejemplos que otorga la información producida a través de las entrevistas es el de la organización de eventos contra el trabajo infantil, la caminata por los Derechos de Infancia o sus similares.

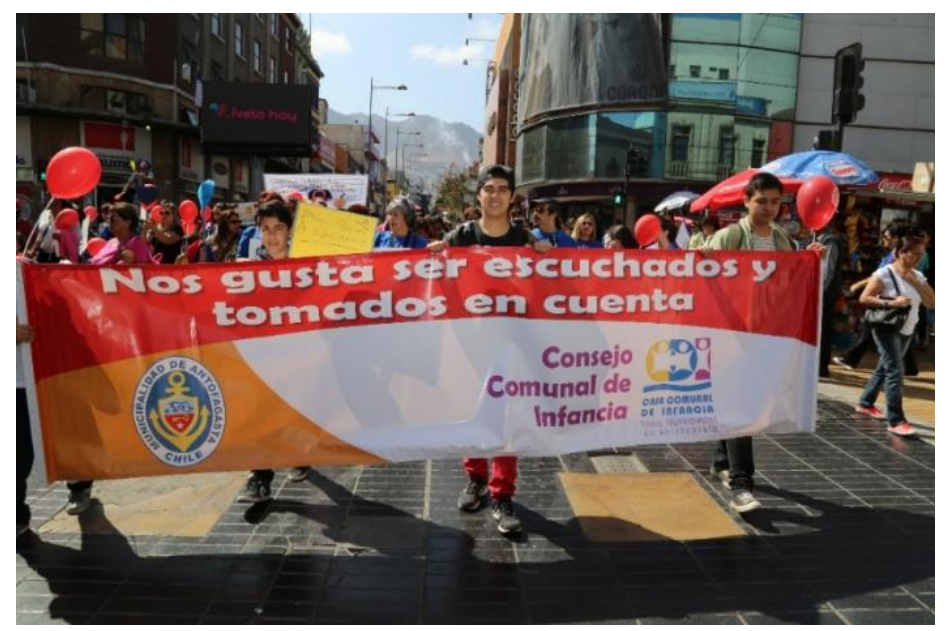

Se destaca como mayor fortaleza del CCI su unidad y cohesión interna, factor que permite que sus liderazgos ejerzan una influencia positiva en los miembros más pequeños del consejo, fomentando una vocación constructiva, el aprendizaje y la sana convivencia fraterna, elemento que por cierto produce una importante mediación en las percepciones que tienen de los cambios que han sufrido los consejeros y exconsejeros con el tiempo tras su participación en el Consejo. Con el mismo énfasis, se tematiza la posibilidad de que el CCI sirva como una escuela de formación para jóvenes, como los próximos líderes políticos de la comuna: aquí existe un punto relevante, el cual tiene que ver con las proyecciones que tienen los distintos 
jóvenes hacia fuera del consejo, para con sus comunidades locales, así como con la madurez con la que sean capaces de enfrentar los desafíos fuera de ésta, como señala el ex consejero Francisco Pastene al comparar la participación en el Consejo con un trabajo:

“(...) entonces de ahí uno se daba cuenta de que verdad es un trabajo, que aunque no sea remunerado era un trabajo que tú lo hacías de cierta manera con mucha vocación, porque por lo general habían niños que, de verdad salían escogidos y les daba lo mismo, no seguían, no tenían el compromiso. Pero yo por lo menos, seguí hasta el final de los tiempos.” (Francisco Pastene, exconsejero 2011)

Además de lo mencionado, también se realzan los valores de representatividad, ecuanimidad, renovación permanente de las personas pero con la mantención de un modo y códigos operativos para velar por el éxito de los procesos y las actividades en cuestión:

"Durante los dos años que he estado, el Consejo Comunal de Infancia se organiza de un encuentro de participación ciudadana infantil y juvenil, los cuales son invitados representantes del Centro de Alumnos de cada colegio tanto municipal, particular y subvencionado y particular pagado, lo que hace también es asegurar la mayor representatividad posible, también se invitan a niños que están fuera del ámbito escolar, como lo son los niños del Hogar de Cristo, niños que están en la Teletón.” (Marcial Valdivia, Consejero Vigente)

En general, los concejeros entrevistados coinciden en tener valoraciones positivas no sólo de sus experiencias particulares en el consejo, sino también de lo que pudieron lograr, así como a todo aquello que pudieron obtener de su participación en éste, y los vínculos y redes (tanto afectivas con sus compañeros, como con las autoridades de la Municipalidad) que establecieron y alimentaron a través de su participación.

\section{Proyecciones sobre la participación en el CCI}

Es importante dar cuenta de que los entrevistados entienden que, dado que su participación a través del consejo es limitada por una barrera etaria, el CCI les ofrece una ventana al mundo, determinando de forma importante sus expectativas de cara a la formación profesional en áreas marcadas por el trabajo colectivo y la constitución del bienestar social como son las 
áreas de salud, educación y justicia, históricamente vinculadas también a la oferta de trabajo en el sector público. Dichas áreas son las más mencionadas por los entrevistados, quienes se identifican con un perfil de formación humanístico-artística y recalcan en su discurso los distintos rasgos críticos interiorizados a partir de los distintos proyectos educativos propios de los planteles que integran.

En el caso de los entrevistados que no recibieron dicha formación formalmente, el elemento que viene a suplirla ha sido justamente el trabajo en el seno del CCI, del cual recalcan su papel en la generación de competencias para el trabajo con comunidades heterogéneas con la centralidad en el territorio. La siguiente cita al ex consejero de 2013 Matías Tobar, por su parte, releva la importancia del compañerismo en dichas labores de coordinación, como en su valoración del CCI y sus relaciones hacia fuera de quiénes lo integran.

"Hubo compañerismo, confianza, eso más que nada hubo en el Consejo en cuanto a lo que yo estuve en ese periodo (2013-2014). Como le vuelvo a reiterar compañerismo, tiene mucho compañerismo, se unen unos con otros, no tienen distinción unos con otros. Se unen, se unen todos en una sola causa. Yo creo que no hay nada que mejorar, porque los elementos están dados a la par con el Consejo y los asesores, los dos dan los elementos.” (Matías Tobar, ex consejero 2013-2014)

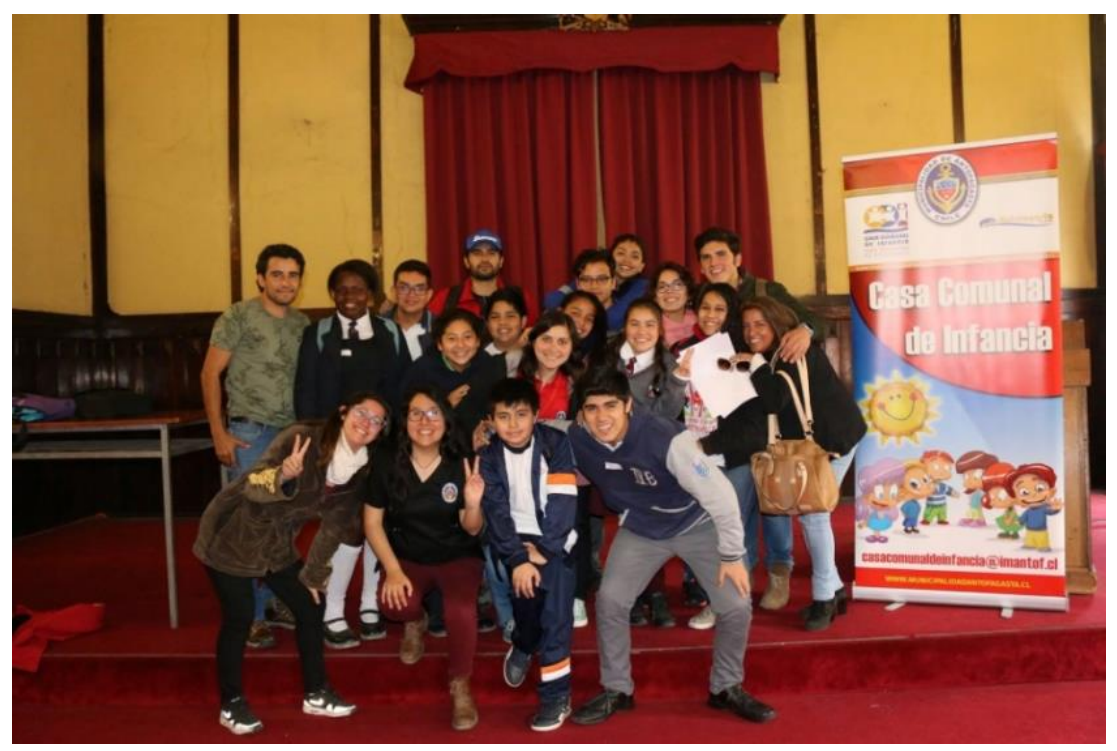


Es sumamente importante también comprender el rol de plataforma socio-afectiva que ha jugado el CCI para sus distintos integrantes. Tanto como una ventana al mundo, como se señala anteriormente, éste les permite reflexionar sobre sí mismos, entenderse en el contexto de producir socialmente sus vidas e intervenir políticamente en los asuntos que les suscitan interés. Muchas de las opiniones de los entrevistados giran justamente en torno a cómo el CCI les ha marcado especialmente en sus expectativas a futuro, así como en la forma que tienen de entender su relación con otros, y cómo se toman el trabajo colectivo con la madurez suficiente al mismo tiempo que han logrado madurar personalmente, como señala Daniela Pumarino en el apartado anterior respecto de su "florecimiento personal" y el papel que jugó el CCI en éste.

Todos estos elementos que dicen relación con su futura inserción en el mercado laboral y la potencial experticia que desarrollan ante los desafíos que implica la intervención en el sector público y la implementación de proyectos en sus territorios.

Uno de los elementos que más se repite, especialmente en el discurso de los consejeros y exconsejeros, tiene que ver con proyectar una transformación en la cantidad de comisiones que componen el consejo, con el objetivo de abarcar una mayor cantidad de problemas específicos de la comunidad, al mismo tiempo que se releva la necesidad de ser escuchados por las autoridades, realizándose cambios sustanciales de acuerdo a las necesidades del consejo. Esto, sin necesariamente descuidar el viraje implícito de la agenda de trabajo sostenida hasta el momento por el CCI hacia los temas que conforman la contingencia nacional. Tobar, por su parte, señala que seguiría participando como mano de obra útil y competente para los eventos públicos del CCI, a raíz de la experiencia adquirida en su colegio.

Más allá de las valoraciones positivas que tienen de su experiencia en el CCI, los consejeros y exconsejeros entrevistados también reportan la necesidad de proponer y mejorar una serie de aspectos de éste.

Por ejemplo, el consejero Manuel del Canto es enfático en proponer un CCI que tenga un papel más activo en recoger las percepciones y conocer lo que opinan los niños fuera del consejo sobre los temas que éste trabaja, esto es, de poder dialogar más directamente con sus 
comunidades (y no exclusivamente a través de quiénes las representan). En sintonía con lo afirmado por Manuel, el otro consejero vigente entrevistado (Marcial Valdivia) señala el hecho de que el carácter de "la voz de los niños" debe ser especialmente pensado y reflexionado por el Consejo, así como por la sociedad y cómo ésta les considera: se trata de visiones honestas, que deben ser representadas y no desacreditadas por las autoridades por el solo hecho de ser niños. Una de sus propuestas, ante esto, es por ejemplo abrir el CCI a ser integrado por una mayor cantidad de personas, suponiendo que éstos podrían ser especialmente de menor edad, así como poner especial énfasis en la difusión del proyecto que es el CCI.

La idea, según Valdivia, se sostiene sobre la necesidad de aclarar las responsabilidades de los adultos sobre los niños, así como también las responsabilidades de éstos sobre sí mismos, y en todo minuto, instituciones como el CCI deben ocupar un papel protagónico en definir dichas cuestiones a nivel societal. Francisco Pastene, por su parte, coincide con el consejero Valdivia en el hecho de que es necesario para mejorar la inserción social del CCI poner un especial énfasis en el desarrollo de una política comunicacional y de difusión que les permita llegar a todos los sectores sociales de la comuna.

\section{Conclusiones}

El trabajo realizado durante estos 8 años ha estado marcado por la necesidad de conformar una comunidad entre los líderes locales que les permita conducir tanto sus experiencias de vida, como las distintas problemáticas que forman parte de su diario vivir, dando pie primero a una reflexión colectiva, y ofreciendo la opción de proyectar en ciclos estructurados la posibilidad de actuar sobre aquellos problemas y transformar la realidad de sus comunidades.

En ese sentido, la participación de los distintos líderes de las comunidades que confluyen en el Consejo no es sólo el motor que hace funcionar al Consejo, sino también el motor para hacer posibles los cambios que este pretende lograr para las comunidades. Como portadores de dicha posibilidad, el protagonismo de consejeros y ex consejeros se sostiene y alimenta por las facilidades entregadas por directivos, tanto como por el quehacer de los profesionales de la Casa Comunal de la Infancia. 
Asimismo, es importante destacar el papel que juega en todo esto el CCI como una plataforma que se comunica y coordina con otras instancias. El cuidado en dar cuenta de su carácter de plataforma radica en la necesidad de hablar de su papel como el espacio en el cual se articulan las percepciones, reflexiones y valoraciones de los NNA de la comuna, así también como una seria de planteamientos, por ejemplo, el cómo hacer más eficiente y eficaz el espacio en su doble papel como formador y vehículo de las transformaciones de los jóvenes para su juventud, cómo instalar la necesidad de la participación y avanzar hacia la constitución de acuerdos con las comunidades educativas y el estamento docente, cómo hacer del servicio público un valor propio de comunidades que avanzan hacia una buena vida colectiva - son todas preguntas que deben formar parte a futuro de la agenda del CCI.

En teoría, los resultados de la investigación nos permiten no sólo dar un espacio en la historia a lo ocurrido en, desde y hacia el Consejo en sus 8 años de existencia, sino que también nos otorgan la posibilidad de entender a niños y jóvenes como algo que rebasa una categoría: al abordar al joven como sujeto, esto es, como el producto estructural y a la vez el agente y actor creador de relaciones sociales hacia el espacio y el tiempo.

Ahora, no se ha tratado de construir tautológicamente la idea de joven y confirmarla a través de un estudio que dé cuenta de sus manifestaciones en la situación local, sino justamente de explicar cómo se ha producido aquel proceso en el pasado reciente: a partir de los testimonios de los distintos actores comprometidos en el estudio, vemos cómo aquel sujeto se enfrenta a una condicionante de su espacio-tiempo como es el carácter adulto-céntrico de la democracia a nivel mundial. Chile, menos aún Antofagasta, son la excepción (y si lo fueran, confirmarían el carácter de regla de dicha aseveración). En dicho escenario, cobra especial relevancia el papel del Consejo, tanto como la práctica de sus integrantes, en la acumulación de un capital político que permite posicionar al CCI como interlocutor hacia las comunidades de sus integrantes tanto como hacia las autoridades municipales y regionales.

En esa línea, las experiencias de la niñez y la juventud cobran sentido no sólo como etapas necesarias del desarrollo biológico de todo aquel ser humano que se precie de conservar sus derechos básicos, sino también como virtudes fundamentales de cualquier sociedad que vele 
por aquellos derechos. Queda instalada de esta forma la pregunta de cómo la ciudad es capaz de observarse a sí misma a través de los ojos de su juventud.

Lo anterior, entonces, prefigura el camino para avanzar hacia la conformación de cada vez más espacios para el ejercicio de una comunidad ciudadana responsable, democrática y empoderada, que asume la niñez como un frente para disputar el ejercicio y la validez de sus derechos, y con ello asume también el desafío de la inclusión como un elemento fundante de su propia práctica, en tanto aquel "nosotros" como ciudadanas y ciudadanos se funda en el respeto a la diversidad de quienes forman parte de la ciudad.

\section{Referencias bibliográficas}

Belmonte, C. (2003). Una ciudad para los niños: políticas locales de infancia. La Mancha: Exlibris.

Benedicto, J. (2003). Infancia y juventud: nuevos sujetos de ciudadanía. Un reto para la sociología de la infancia. Red por los Derechos de la Infancia en México. Recuperado de http://www.derechosinfancia.org.mx/Temas/temas_sociologia2.htm

Bloj, C. (2015). Ciudades e infancia. Juego, participación y derechos culturales en Rosario (Argentina). CEPAL, Serie Políticas Sociales. Recuperado de http://repositorio.cepal.org/bitstream/handle/11362/37956/1/S1500247_es.pdf

Canales, M; Ghiardo, F. y Opazo, A. (2015). Para un concepto de juventud. En Juventudes: metáforas del Chile contemporáneo. Pablo Cottett Editor.

Corvera, N. (2011). Participación ciudadana de los niños como sujetos de derechos. Persona y Sociedad, 2 (25), 73-99.

Diestro, A. (2008). La ciudad de los niños. Una experiencia político pedagógica en perspectiva comparada. En XI Congreso de Educación Comparada. La educación como respuesta a la diversidad. Sevilla, mayo 2008.

Duarte, K. (2011). Vulnerabilidad y vulneración de derechos de los niños, niñas y jóvenes en contextos adultocéntricos. En Vulnerabilidad y derechos en la niñez y juventud chilena. Santiago de Chile: Caritas Chile. 
Duarte Quapper, Claudio. (2012). Sociedades adultocéntricas: sobre sus orígenes y reproducción. Última década, 20(36), 99-125. https://dx.doi.org/10.4067/S071822362012000100005

Franco, F., Mir, A. \& Moljo, F. (2009). Ciudades Amigas de la Infancia: Aproximaciones a las políticas sociales "amigables" con la infancia de las ciudades de Rosario y Buenos Aires. Especialización y Maestría en Derechos Humanos y Políticas Sociales, trabajo final de integración. Universidad Nacional de San Martín, Buenos Aires. Recuperado de http://www.equidadparalainfancia.org/wpcontent/uploads/2013/01/ciudades_amigas_de_la_infancia.pdf

Giorgi, V. (2010). La participación infantil de niños, niñas y adolescentes en las Américas. OEA - Instituto Interamericano del Niño, la Niña y Adolescentes. Recuperado de http://www.iin.oea.org/iin/Pdf/publiclibrilloESPA\%C3\%910L.pdf

Guilló, J. (2003). Una ciudad también para los niños. En C. Belmonte (Ed.), Una ciudad para los niños: políticas locales de infancia (pp. 33-40). La Mancha: Exlibris.

James, A., Prout, Al. (Eds.) (2010 [1997]). Constructing and Reconstructing Childhood. Contemporary Issues in the Sociological Study of Childhood. Oxon: Routledge.

Liebel, M. \& Martínez, M. (2009). Infancia y derechos humanos: hacia una ciudadanía participante y protagónica. Lima: IFEJANT, Instituto de Formación para Educadores de Jóvenes, Adolescentes y Niños Trabajadores de América Latina y El Caribe.

Moneti, F. (2008). ¿Cómo es una ciudad amiga de la niñez? En La Revista, Red de Información sobre los Derechos del Niño, $\mathrm{N}^{\circ} 22$.

Pavez, I. (2012). Sociología de la Infancia: Las niñas y los niños como actores sociales. Revista de Sociología, 27, pp. 81-102.

Rojas Flores, J. (2010). Historia de la infancia en el Chile republicano, 1810-2010. Santiago, Chile: World Color.

UNICEF (2004). Construyendo ciudades amigas de la infancia. Un marco de Acción. Centro de Investigaciones Innocenti, Secretariado Internacional para ciudades amigas de la 
Infancia, Educación y Aprendizaje (IEYA). Vol. 6, № 2, pp. 85-109. ISSN: 0719-6202 http://revistas.uv.cl/index.php/IEYA/index

Infancia,

Florencia.

Recuperado

de

http://www.unicef.es/sites/www.unicef.es/files/Construy_CAI.pdf 